\title{
Analysis of Subcellular Prefoldin 1 Redistribution During Rabies Virus Infection
}

\author{
Jinyang Zhang ${ }^{1}$; Qinqin Han $^{1}$; Yuzhu Song ${ }^{1}$; Qiang Chen ${ }^{1}$; Xueshan Xia ${ }^{1,}$ \\ ${ }^{1}$ Research Center of Molecular Medicine of Yunnan Province, Faculty of Life Science and Technology, Kunming University of Science and Technology, Kunming, China \\ ${ }^{*}$ Corresponding author: Xueshan Xia, Research Center of Molecular Medicine of Yunnan Province, Faculty of Life Science and Technology, Kunming University of Science and Technol- \\ ogy, P. O. Box: 650500, Kunming, China. Tel: +86-87165939528, Fax: +86-87165939528, E-mail: oliverxia2000@aliyun.com
}

Received: October 23, 2014; Revised: January 17, 2015; Accepted: February 9, 2015

\begin{abstract}
Background: Rabies virus (RABV) is one of the old deadly zoonotic viruses. It attacks the central nervous system and causes acute encephalitis in humans and animals. Host factors are known to be essential for virus infection and replication in cells. The identification of the key host factors required for RABV infection may provide important information on RABV replication and may provide new potential targets for RABV drug discovery.

Objectives:This study aimed to investigate the change in the subcellular distribution and expression of the host protein Prefoldin subunit 1(PFDN1) in RABV-infected cells and the viral expression of plasmids in the transfected cells.

Materials and Methods: Mouse Neuro-2a (N2a)cells were infected by RABV or transfected with the plasmids of the nucleoprotein (N)and/ or phosphoprotein (P) gene of RABV. The subcellular distribution of PFDN1 was analyzed by confocal microscopy, and the transcription levels of PFDN1 in the N and/or P gene of the RABV-transfected or RABV-infected N2a cells were assessed via real-time quantitative polymerase chain reaction.

Results: Confocal microscopy showed that PFDN1 was colocalized with the $\mathrm{N}$ protein of RABV in the infected N2a cells and was mainly recruited to the characteristic Negri-Body-Like(NBL) structures in the cytoplasm, as well as the cotransfection of the N and P genes of RABV. The transcription of PFDN1 in the RABV-infected N2a cells was upregulated, whereas the transfection of the N and/or Pgenes did not result in the upregulation of PFDN1.

Conclusions: The results of this work demonstrated that the subcellular distribution of PFDN1 was altered in the RABV-infected N2a cells and colocalized with the N protein of RABV in the NBL structures.
\end{abstract}

Keywords: Prefoldin; Redistribution; Rabies Virus

\section{Background}

The highly neurotropic Rabies virus (RABV) is the most significant human pathogen of the Rhabdoviridae family and is a prototype member of the Lyssavirus genus. RABV is the causative agent of rabies, which remains a global public problem with an estimated human death toll of more than 55000 annually (1). The RNA genome $(\sim 12 \mathrm{~kb})$ of RABV encodes five viral proteins, including Nucleoprotein (N), Glycoprotein (G), Phosphoprotein (P), Matrix protein (M), and RNA-dependent RNA polymerase (L). It has been shown that the transcription and replication of RABV take place within Negri-Body-Like (NBL) structures, which are inclusion bodies formed in the process of RABV infection (2). The NBL structures of RABV-infected cells are composed of the viral nucleocapsid, viral genome, and a series of cellular proteins (3).

Prefoldin binds specifically to cytosolic chaperonins (e.g. chaperonin TRiC/CCT) and transfers the target proteins to it. Prefoldin binds to the nascent polypeptide chain and promotes folding in an environment in which there are many competing pathways for nonnative pro- teins. Prefoldin subunit 1 (PFDN1) was shown to be one of the differential proteins in the Vaccinia virus IHD-W infected HEK 293 cells (4). The hepatitis C virus (HCV) F protein was found to interact with cellular protein PFDN2 through the use of the yeast two-hybrid system and perturb the tubulin cytoskeleton organization (5). PFDN1 and PFDN2 are two of the upregulated and downregulated differentially expressed proteins between a hepatitis B virus (HBV)-producing cell line HepG2.2.15 and its parental cell line HepG2, respectively.

Viruses are fully reliant on the successful recruitment of host cellular factors in their viral life cycle, and viruses usually seize control of the host signaling pathways and cellular translation factors, which regulate their activity (6). Host protein Hsp70 is present in both purified RABV virions and in nucleocapsids purified from infected cells, and it also has been shown to interact with the $\mathrm{N}$ protein of RABV (7). Dynein light chain 8 (DLC8) has been reported to bind to the P protein of RABV and promote efficient viral transcription (8-10). The $\mathrm{M}$ of RABV plays a role in

Copyright (C) 2015, Ahvaz Jundishapur University of Medical Sciences. This is an open-access article distributed under the terms of the Creative Commons Attribution-NonCommercial 4.0 International License (http://creativecommons.org/licenses/by-nc/4.0/) which permits copy and redistribute the material just in noncommercial usages, provided the original work is properly cited. 
inhibiting translation in virus-infected cells through an interaction with the eukaryotic translation initiation factor 3, subunit H (eIF3h) (11). Recently, we found out that the subcellular distribution of host proteins CCT $\gamma$ (3) and CCT $\alpha$ (12) was altered in RABV-infected cells and that it played a role in RABV infection. However, we detected no direct interaction between CCT $\gamma$ or CCT $\alpha$ and the viral proteins $\mathrm{N}$ or $P$. We are, therefore, still in the dark as to whether the upstream of chaperonin CCT, Prefoldin, has a similar subcellular redistribution in RABV-infected Neuro-2a (N2a) cells.

\section{Objectives}

Besides viral proteins, host cellular factors play a key role in the replication of various viruses. The aim of the present study was to investigate the subcellular distribution and transcription of the chaperone PFDN1 in RABVinfected N2a cells.

\section{Materials and Methods}

\subsection{Cell Culture, Viruses, Antibodies, and Reagents}

Mouse N2a cells were grown at $37^{\circ} \mathrm{C}$ in a humidified $\mathrm{CO}_{2}$ (5\%) incubator with completed Dulbecco's modified eagle's medium (DMEM) (Invitrogen, CA, USA) supplemented with $10 \%(V / V)$ fresh newborn calf serum (Sijiqing, Hangzhou, China). The RABV HEP-Flury strain was propagated in N2a cell monolayers. Rabbit polyclonal Antibody (pAb) against PFDN1 was purchased from ProteinTech Group Inc. (Chicago, IL, USA), Rabbit monoclonal Antibody (mAb) against DLC8 was purchased from Epitomics Inc. (Burlingame, CA, USA), and 4', 6-diamidino-2-phenylindole (DAPI) was purchased from Sigma-Aldrich (St. Louis, MO, USA). Mouse mAb anti-N protein of RABV was produced as described previously $(3,13)$.

\subsection{Transfection}

One day before transfection, the cells were plated at a density of $10^{4}$ per well containing a $10 \%$ fetal calf serum DMEM without antibiotics. On the day of transfection, the culture should have $80 \%$ confluency. The pCI-neo-N and pCI-neo-P vectors were cotransfected or monotransfected into the N2a cells using Lipofectamine 2000 (Invitrogen, USA). Forty-eight hours later, the cells were fixed with ice-cold acetone-methanol (1/1) for subsequent use in immunofluorescence or directly extracted RNA for real-time quantitative polymerase chain reaction (qPCR).

\subsection{Co-Focal Microscopy Assay}

The mouse N2a cells were grown in a 96-well plate to $80 \%$ - 90\% confluence before being infected with RABV at a multiplicity of infection (MOI) of 1. Cultured for 48 hours, the cells were washed with phosphate-buffered saline, fixed with pre-chilled acetone-methanol (1/1) at $20^{\circ} \mathrm{C}$ for $20 \mathrm{~min}$, and air dried. After having been blocked by $5 \%$ skimmed milk, the cells were incubated with mouse mAb against the $\mathrm{N}$ gene of RABV and rabbit pAb against PFDN1 or rabbit mAb against DLC8 for 2 hours. The nuclei of the cells were stained with DAPI for immunofluorescence assay.

\subsection{Real-Time Quantitative Polymerase Chain Re- action}

To investigate whether the transcription of PFDN1 was upregulated or downregulated in the RABV-infected and the $\mathrm{N}$ and/or $P$ gene of the RABV-transfected N2a cells, the total RNA of the N2a cells was infected with RABV or transfected with the N or $P$ gene for 48 hours. Also, cDNA synthesis was performed using oligo (dT) primers (100 ng) with a RevertAid first strand cDNA synthesis kit (Fermentas, Thermo Scientific), and real-time qPCR was performed as described previously (3). Briefly, it was carried out on the 7500 real-time PCR system with $200 \mathrm{ng}$ of cDNA template, $1 \times$ SYBR premix Ex taq (perfect real time, TaKaRa, Dalian, China), and $200 \mathrm{nM}$ of each primer in the volume of $20 \mu \mathrm{L}$. Specific primers (Table 1) were designed and synthesized by Shanghai Shenggong (Shanghai, China) to simultaneously amplify various target genes. The amplification conditions were as follows: $95^{\circ} \mathrm{C}$ for $10 \mathrm{~min}$; followed by 40 cycles of $95^{\circ} \mathrm{C}$ for 10 seconds, $60^{\circ} \mathrm{C}$ for 30 seconds, and $72^{\circ} \mathrm{C}$ for 10 seconds. The melting curve was recorded after the PCR was run. And relative expression was calculated by the comparative $\Delta \Delta \mathrm{Ct}$ method.

\section{Results}

\subsection{Subcellular Redistribution of Prefoldin Subunit 1 and Dynein Light Chain 8 in the Rabies Virus-In- fected Neuro-2a Cells}

Given the similarity between chaperone complex Prefoldin and chaperonin complex TRiC/CCT, as well as their cooperation in protein folding, the question is whether an alteration of Prefoldin distribution occurred in the RABV-infected N2a cells. DLC8 has been reported previously by several research teams to be a partner of the $\mathrm{P}$ gene of RABV and used as a positive control. Immunostaining was conducted using $\mathrm{mAb}$ to the $\mathrm{N}$ protein of RABV, followed by Tetramethylrhodamine (TRITC)conjugated IgG (red fluorescence) and counterstaining using rabbit antibodies to PFDN1 and DLC8, followed by FITC-conjugated IgG (green fluorescence). The cell nuclei were stained with DAPI (blue fluorescence). The triplestained cells were observed by Leica TCS SP5 laser confocal microscopy. The relevance of PFDN1 and DLC8 to the localization of the $\mathrm{N}$ protein of RABV was analyzed by confocal microscopy (Figure $1 \mathrm{~A}$ and $2 \mathrm{~B}$ and Figure $2 \mathrm{~A}$ and $2 \mathrm{~B}$ ). In the RABV-infected N2a cells, the neuroblastomas (NBs) containing the viral $\mathrm{N}$ gene in the cytoplasm was colocalized with intracellular protein PFDN1 (Fig- 
ure $1 \mathrm{~A}$ ) and DLC8 (Figure $2 \mathrm{~A}$ ). In the mock-infected N2a cells, PFDN1 was distributed both in the nucleus and cytoplasm; however, PFDN1 was mainly distributed in the cytoplasm of the N2a cells (Figure 1 B), while DLC8 was homogeneously distributed in the cytoplasm and nucleus of the N2a cells (Figure $2 \mathrm{~B}$ ).

\subsection{Transcriptional Alterations of Prefoldin Sub-} unit 1 and Dynein Light Chain during Rabies virus Infection

To determine the change of PFDN1 and DLC8 in tran- scriptional levels, the genes encoding PFDN1 and DLC8 were quantified by comparative real-time PCR (Figure 3) using primers shown in Table 1. The mRNA transcript of glyceraldehyde 3-phosphate dehydrogenase (GAPDH) was used as an internal control housekeeping gene. Both of the two proteins are upregulated in varying degrees. The transcript abundance of PFDN1 in the RABV-infected N2a cells was raised 1.23 times as against the abundance of the mock-infected N2a cells, whereas the transcript abundance of DLC8 in the RABV-infected N2a cells was increased fivefold as compared with the abundance of DLC8 in the mock-infected N2a cells.

\begin{tabular}{|c|c|c|}
\hline Gene Symbol & Forward Primers (5' $\rightarrow$ 3') & Reverse Primers $\left(5^{\prime} \rightarrow 3^{\prime}\right)$ \\
\hline GAPDH & TCAACAGCAACTCCCACTCTTCCA & ACCCTGTTGCTGTAGCCGTATTCA \\
\hline DLC8 & TGTCGGAAGAGATGCAACAGGACT & ATGGGCCGCAATATCCTTCTCGAT \\
\hline PFDN1 & AGGTCCCAACTGAATGTCTGCCAA & AGGCTTGGACAGCTTTCTCCATCT \\
\hline
\end{tabular}

\footnotetext{
a Abbreviations: Dynein light chain 8; GAPDH, Glyceraldehyde 3-phosphate dehydrogenase; DLC8, PFDN1, Prefoldin subunit 1.
}

Figure 1. Subcellular Distribution of Prefoldin Subunit 1(PFDN1) in the Rabies Virus (RABV)-Infected and Mock-Infected Neuro-2a Cells

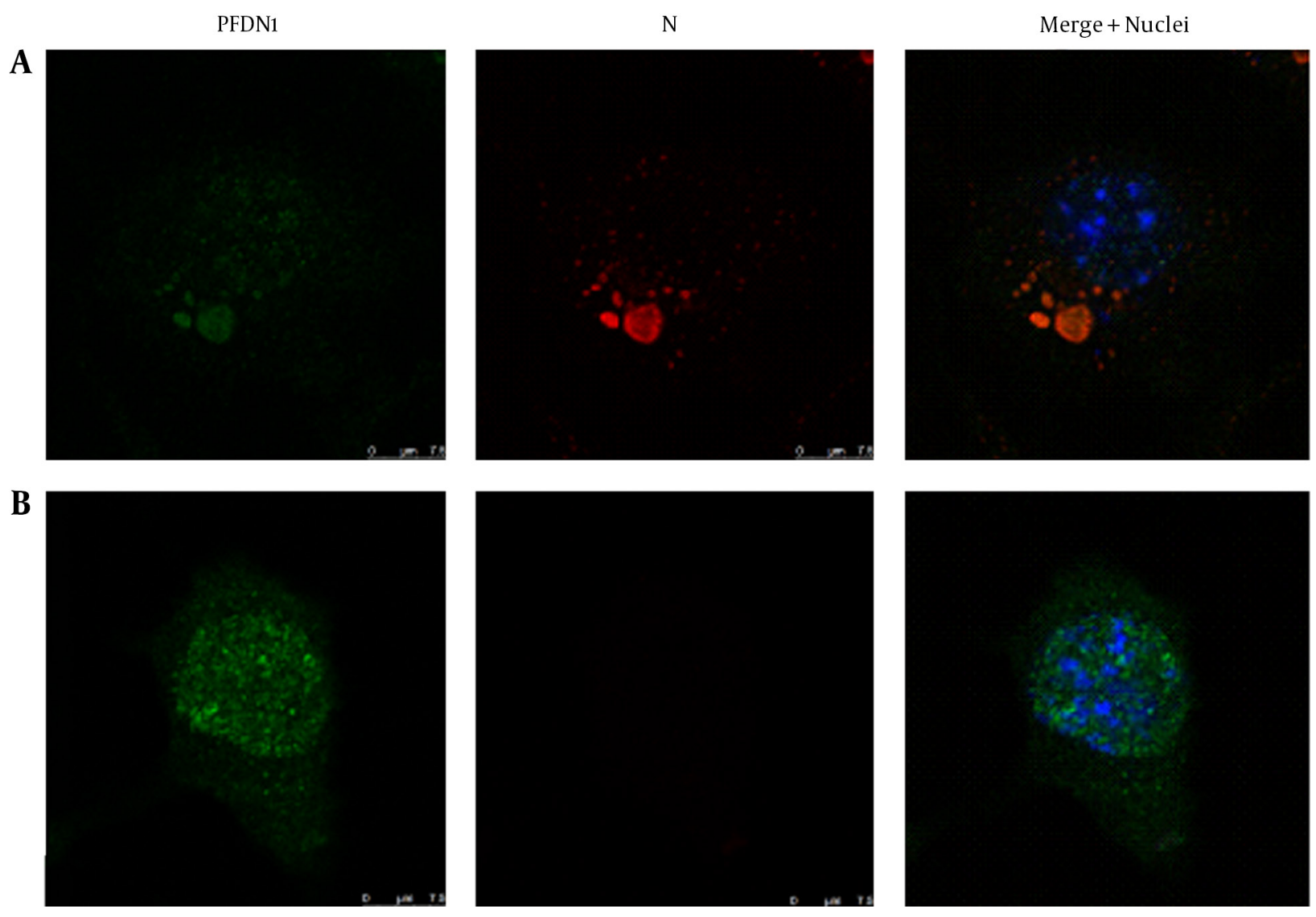

Nuclei are shown in blue, PFDN1 in green, and the N protein of RABV in red. A, RABV-infected cells; B, Mock-infected cells. Scale bars $=7.5 \mu \mathrm{m}$. 
Figure 2. Subcellular Distribution of Dynein Light Chain 8 (DLC8) in the Rabies Virus (RABV)-Infected and Mock-Infected Neuro-2a Cells
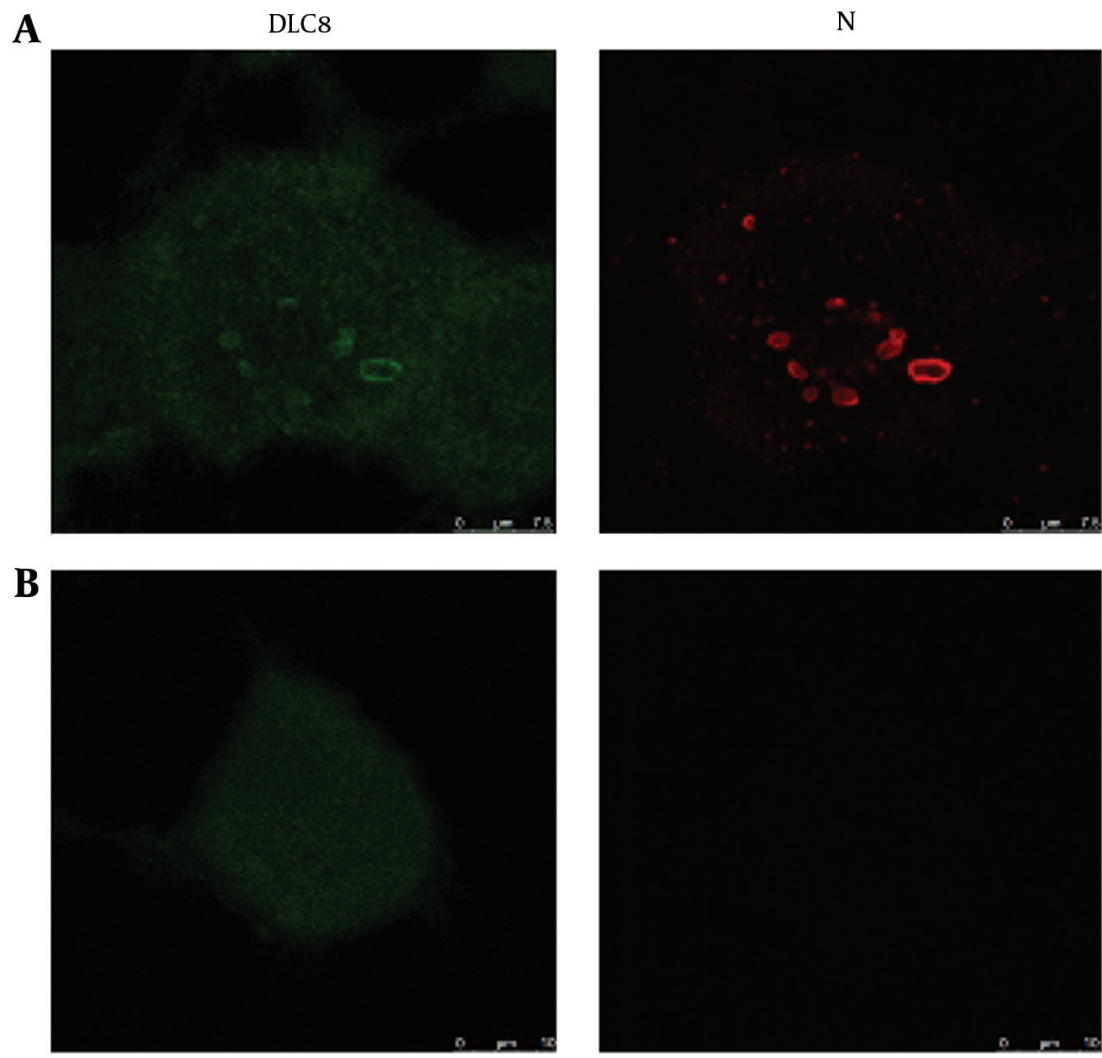

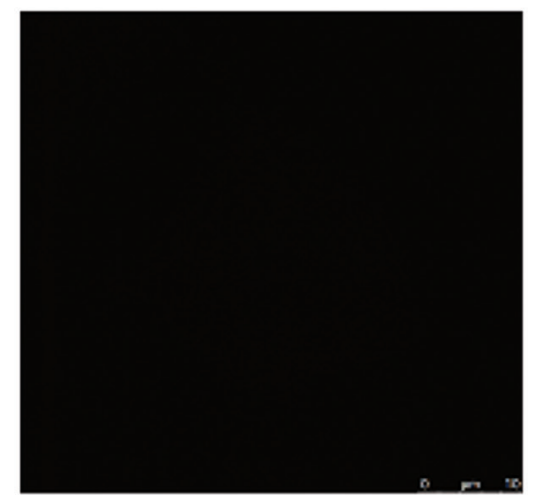

Blue, green, and red fluorescence reflects viable nuclei, host protein DLC8, and N protein of RABV, respectively. A, RABV-infected cells; B, Mock-infected cells. Scale bars $=7.5 \mu \mathrm{m}$ or $10 \mu \mathrm{m}$.

Figure 3. Analysis of Host Genes in Transcriptional Levels in the Rabies Virus-Infected Cells

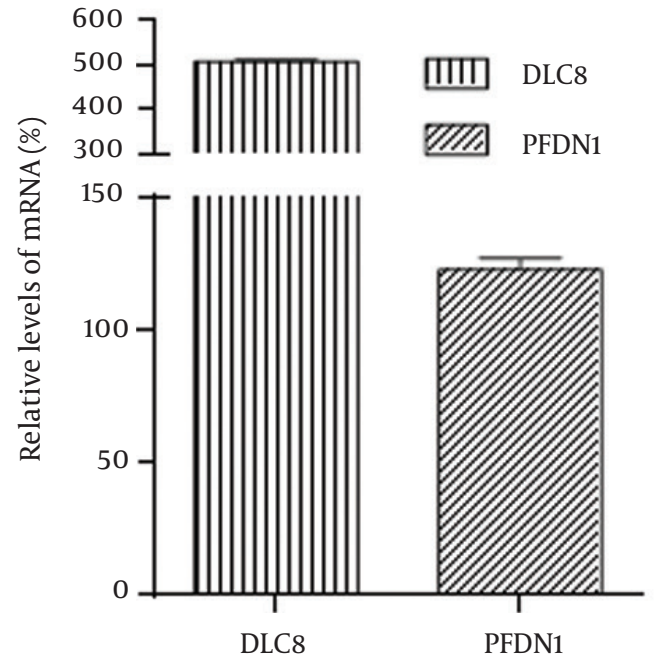

The transcripts of the genes were tested by comparative real-time qPCR and GAPDH was used as internal control. The data are presented as a percent of the N2a cells mock infected in the parallel control set as 100\%.
4.3. Co-Localization of Prefoldin Subunit 1 and Dynein Light Chain 8 with Negri-Body-Like Structures in the Plasmids Nucleoprotein- and Phosphoprotein-Cotransfected Cells

We have previously demonstrated the re-localization of CCT $\gamma$ in cotransfected $\mathrm{N}$ and P genes of RABV in N2a cells (3). To determine whether proteins PFDN1 and DLC8 were involved in the formation of NBL structures, we used immunofluorescence to check the subcellular distribution of the PFDN1, DLC8, and N gene of RABV in pCI-neo-N and pCI-neo-P cotransfected cells. In the $\mathrm{N}$ and $P$ cotransfected N2a cells, the NBL structures were formed, which induced the co-localization of PFDN1 and DLC8 with the N protein of the NBL structures (Figure 4). These results imply that PFDN1 and DLC8 are directly or indirectly involved in the formation of Negri bodies at early stages.

\subsection{Transcriptional Alterations of Prefoldin Sub-} unit 1 and Dynein Light Chain 8 in the PlasmidTransfected Cells

We next investigated whether the transfection of the $\mathrm{N}$ and $\mathrm{P}$ genes of RABV individually or their cotransfection could induce the upregulation of the PFDN1 and DLC8 in 
Figure 4. Co-Localization of the Rabies Virus (RABV) Nucleoprotein with Dynein Light Chain 8 (DLC8) and Prefoldin Subunit 1 (PFDN1) in Plasmids Nucleoprotein- and Phosphoprotein-Co-Transfected Neuro-2a Cells

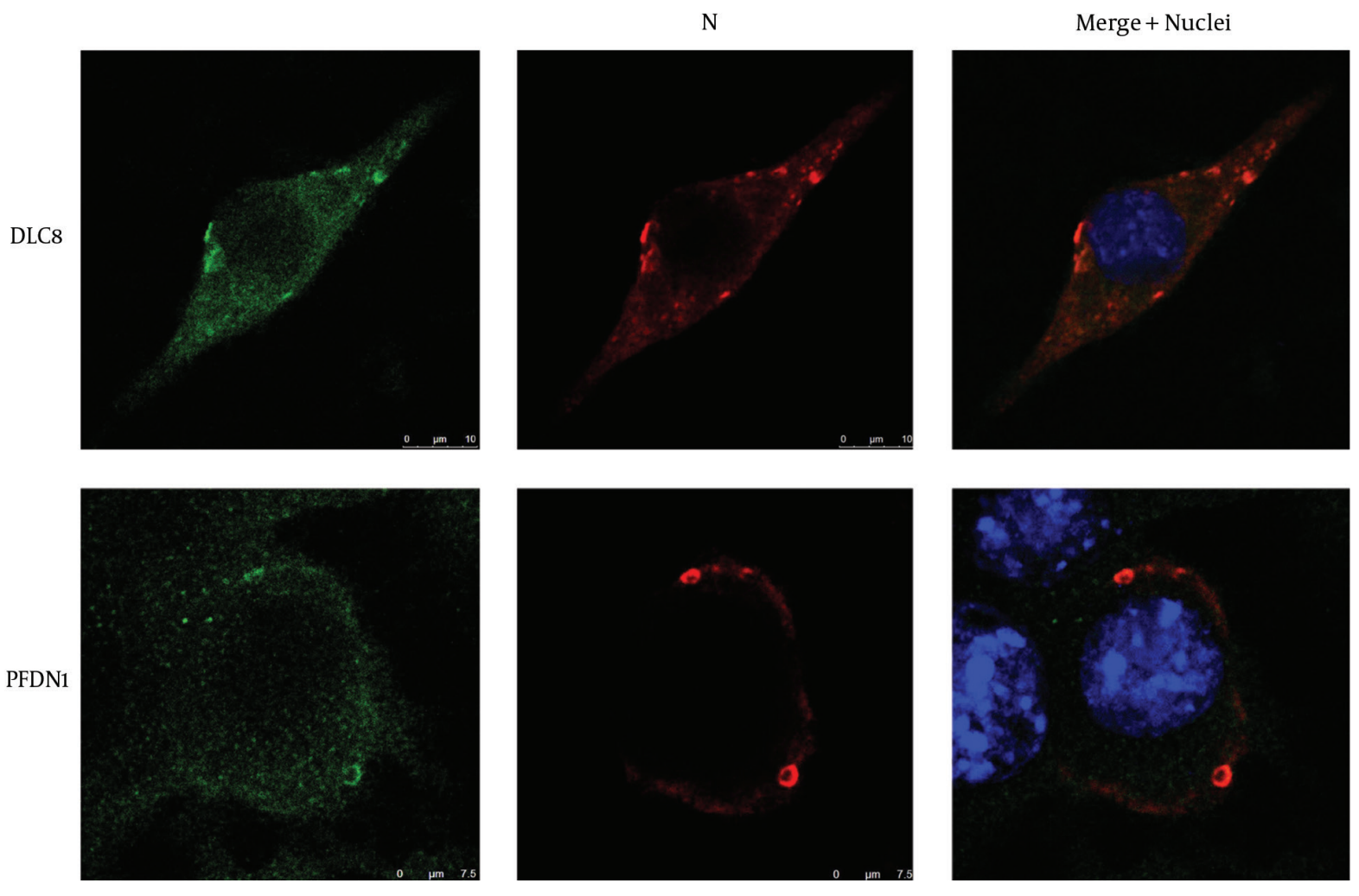

Nuclei (blue) were stained with 4', 6-diamidino-2-phenylindole (DAPI). PFDN1 and DLC8 are in green and the N protein of RABV in red. Scale bars $=7.5 \mu \mathrm{m}$ or $10 \mu \mathrm{m}$.

the N2a cells. The mRNA transcript of PFDN1 and DLC8 was quantified by comparative qPCR. The gene transcriptional levels of the PFDN1 and DLC8 in the pCI-neotransfected N2a cells were normalized to $100 \%$. Compared with the pCI-neo-transfected cells, both of the cells monotransfected with plasmid pCI-neo-P and cotransfected with plasmid pCI-neo-N were able to induce the upregulation of DLC 8 by $38.78 \%$ and $13.98 \%$, respectively (Figure 5). Nevertheless, the transfection had no significant effect on the transcript of PFDN1 (Figure 5). Additionally, compared with the non-transfected cells, our results showed that the transfection itself was able to induce the host genes' upregulation at vary levels, as is shown in Figure 5.

\section{Discussion}

In this study, we used co-focal laser microscopy and real-time qPCR to investigate the alterations of subcellular distribution and transcription of host cellular factors of PFDN1 and DLC8 in RABV-infected and plasmids containing the $\mathrm{N}$ and $\mathrm{P}$ genes of RABV-transfected N2a cells. In two previous studies $(3,12)$, we determined that the chaperonins CCT $\gamma$ and CCT $\alpha$ were re-localized to the NBL structures of RABV-infected and transfected cells,
Figure 5. Analysis of the Host Genes in Transcriptional Levels in the Rabies Virus Nucleoprotein- and/or Phosphoprotein-Transfected Neuro2a Cells
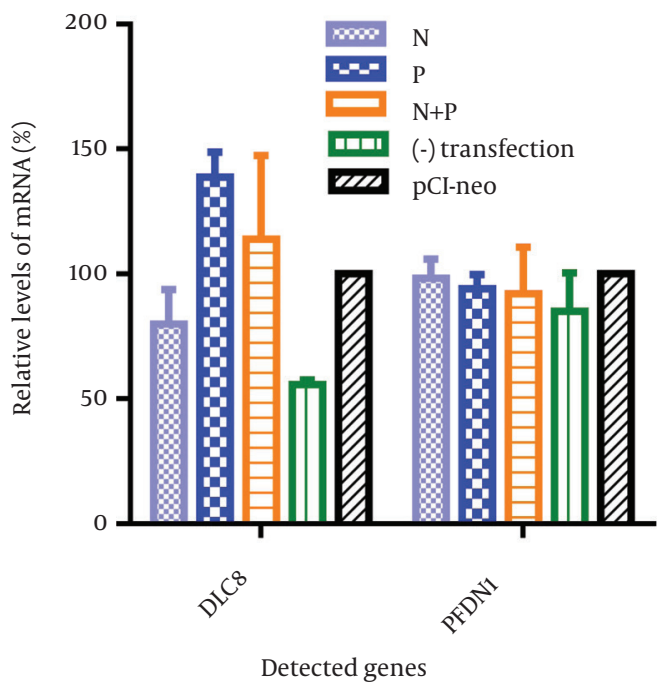

The N2a cells were monotransfected ( $\mathrm{N}$ and $\mathrm{P}$ ) or cotransfected with the $\mathrm{N}$ and $\mathrm{P}$ genes of RABV $(\mathrm{N}+\mathrm{P})$.The transfection of the empty expression vector (pCI-neo) and non-transfected cells (-) was set as control. 
which implies that the chaperone may play a role in the life cycle of RABV. However, we observed no direct interaction between the host protein of CCT $\alpha$ and CCT $\gamma$ and the $\mathrm{N}$ or P protein of RABV through co-immunoprecipitation. This finding seems to be in consequence of an indirect interaction between the up or downstream of TRiC/CCT and viral proteins. It is well accepted that PFDN1 plays an important role in the correct folding of other proteins. It binds specifically to cytosolic chaperonins and transfers the target proteins. The results of the present study were exactly as we had predicted: PFDN1 was redistributed to the NBs in the RABV-infected N2a cells. Although we do not have experimental evidence to explain this phenomenon, given the accumulation of PFDN1 in the cytoplasm NBs observed upon infection, it is tempting to suggest that the virus may have triggered the stress-response protein of the cell against unfolded proteins.

We then asked whether the relocalization of PFDN1 and DLC8 to the cytoplasm NBs structures in the RABVinfected cells was due to the presence of one or two viral proteins of RABV. It should be mentioned that similar immunofluorescence tests were carried out on RABV infection and the plasmids of the RABV-transfected N2a cells. Furthermore, the co-expression of the $\mathrm{N}$ and $\mathrm{P}$ genes of RABV is a key factor for inducing the redistribution of PFDN1 and DLC8. All viruses are dependent on the host cell machinery for their replication and as such modify a series of cellular pathways (14). Remarkably, the results of the current study revealed an increase in the transcript abundance of PFDN1 and DLC8 in the RABV-infected cells. In order to find the viral factor that induced the upregulation of PFDN1 and DLC8, we tested the transcript of the host genes in the $\mathrm{N}$ and P genes of the RABV-transfected N2a cells. The results were interesting inasmuch as the co-expression of the $\mathrm{N}$ and $\mathrm{P}$ genes of RABV was able to induce the upregulation of DLC8, while PFDN1 was not affected significantly. A possible explanation is that probably the minor upregulation of PFDN1 in the RABV-infected cells was induced by the presence of the other proteins of RABV such as G, M, and L. The protein complex of Prefoldin was also found to be upregulated in the brains of patients with Alzheimer's disease (15).

Prefoldin is a jellyfish-shaped heterohexameric co-chaperone of group II chaperonins and is known to assist in capturing protein-folding intermediates and transferring them to group II chaperonins for the completion of folding (16). The manner in which Prefoldin cooperates with the chaperonin and chaperone and interacts with its substrates is poorly understood. Chaperone HSP70 has numerous co-chaperones that support its chaperone function in protein folding. Prefoldin is also one important group of co-chaperones of HSP70 (17). Previously published data by our group showed that the subcellular redistribution of CCT $\gamma$ and CCT $\alpha$ played a role in RABV replication $(3,12)$. In addition, Lahaye et al. (7) reported that HSP70 interacts with the N protein of RABV and suggested that a variety of host chaperonins and chaperones are involved in the replication of RABV. Indeed, Prefoldin is an intermediary factor between HSP70 and TRiC/CCT (17). This could explain why we failed to confirm the interactions between the viral proteins of RABV and CCT.

We speculate that the recruitment of PFDN1 to NBs could also potentially assist in the folding of a variety of viral proteins or host proteins from their nascent polypeptides during the replication of the virus. Our findings provided evidence that upon RABV infection, another chaperone, PFDN1, is redistributed to the cytoplasm NBs, where the interacting partners of the viral replication complexes (i.e. viral RNA N, P, and L) localize.

\section{Acknowledgements}

The authors would like to thank Mrs. Chunmei Meng for technical assistance with laser confocal microscopy.

\section{Authors' Contributions}

Jinyang Zhang carried out most of the experiments, developed the original idea, and wrote the manuscript. Xueshan Xia critically revised the manuscript. Qinqin Han, Yuzhu Song, and Qiang Chen helped with the experiments and material preparation. All the authors approved the final manuscript.

\section{Funding/Support}

This work was supported by the applied basic research projects of Yunnan province (KKS0201326002), Fund for fostering talents of Kunming university of science and technology (KKSY201326085) and construction of science and technology Innovation platform of Yunnan province (2012DA002).

\section{References}

1. World Health Organization . W. Rabies. In: World Health Organization, editor. Geneva, Switzerland: 2013.

2. Lahaye X, Vidy A, Pomier C, Obiang L, Harper F, Gaudin Y, et al. Functional characterization of Negri bodies (NBs) in rabies virus-infected cells: Evidence that NBs are sites of viral transcription and replication. JVirol. 2009;83(16):7948-58.

3. Zhang J, Wu X, Zan J, Wu Y, Ye C, Ruan X, et al. Cellular chaperonin CCTgamma contributes to rabies virus replication during infection. J Virol. 2013;87(13):7608-21.

4. Bartel S, Doellinger J, Darsow K, Bourquain D, Buchholz R, Nitsche A, et al. Proteome analysis of vaccinia virus IHD-W-infected HEK 293 cells with 2-dimensional gel electrophoresis and MALDI-PSDTOF MS of on solid phase support N-terminally sulfonated peptides. Virol J. 2011;8:380.

5. Tsao ML, Chao CH, Yeh CT. Interaction of hepatitis $\mathrm{C}$ virus F protein with prefoldin 2 perturbs tubulin cytoskeleton organization. Biochem Biophys Res Commun. 2006;348(1):271-7.

6. Walsh D, Mohr I. Viral subversion of the host protein synthesis machinery. Nat Rev Microbiol. 2011;9(12):860-75.

7. Lahaye X, Vidy A, Fouquet B, Blondel D. Hsp70 protein positively regulates rabies virus infection. JVirol. 2012;86(9):4743-51.

8. Raux H, Flamand A, Blondel D. Interaction of the rabies virus $\mathrm{P}$ protein with the LC8 dynein light chain. J Virol. 2000;74(21):10212-6.

9. Tan GS, Preuss MA, Williams JC, Schnell MJ. The dynein light chain 8 binding motif of rabies virus phosphoprotein promotes efficient viral transcription. Proc Natl Acad Sci U S A. 
2007;104(17):7229-34.

10. Jacob Y, Badrane H, Ceccaldi PE, Tordo N. Cytoplasmic dynein LC8 interacts with lyssavirus phosphoprotein. J Virol. 2000;74(21):10217-22.

11. Komarova AV, Real E, Borman AM, Brocard M, England P, Tordo $\mathrm{N}$, et al. Rabies virus matrix protein interplay with eIF3, new insights into rabies virus pathogenesis. Nucleic Acids Res. 2007;35(5):1522-32.

12. Zhang J, Ye C, Ruan X, Zan J, Xu Y, Liao M, et al. The chaperonin CCTalpha is required for efficient transcription and replication of rabies virus. Microbiol Immunol. 2014;58(10):590-9.

13. Zhang J, Ruan X, Zan J, Zheng X, Yan Y, Liao M, et al. Efficient generation of monoclonal antibodies against major structural proteins of rabies virus with suckling mouse brain antigen. Monoclon Antib Immunodiagn Immunother. 2014;33(2):94-100.
14. Lawrence P, Rieder E. Identification of RNA helicase A as a new host factor in the replication cycle of foot-and-mouth disease virus. J Virol. 2009;83(21):11356-66.

15. Loring JF, Wen X, Lee JM, Seilhamer J, Somogyi R. A gene expression profile of Alzheimer's disease. DNA Cell Biol. 2001;20(11):683-95.

16. Okochi M, Nomura T, Zako T, Arakawa T, Iizuka R, Ueda H, et al. Kinetics and binding sites for interaction of the prefoldin with a group II chaperonin: contiguous non-native substrate and chaperonin binding sites in the archaeal prefoldin. J Biol Chem. 2004;279(30):31788-95.

17. Broer L, Ikram MA, Schuur M, DeStefano AL, Bis JC, Liu F, et al. Association of HSP70 and its co-chaperones with Alzheimer's disease. J Alzheimers Dis. 2011;25(1):93-102. 